ESAIM: PROCEEDINGS, June 2008, Vol. 23, p. 66-77

L. Boudin, C. Grandmont, Y. Maday, B. Maury, B. Sapoval \& J.-F. Gerbeau, Editors

\title{
A 3D DISCRETE MODEL OF THE DIAPHRAGM AND HUMAN TRUNK*
}

\author{
Emmanuel Promayon ${ }^{1}$ AND Pierre Baconnier ${ }^{1,2}$
}

\begin{abstract}
In this paper, a 3D discrete model is presented to model the movements of the trunk during breathing. In this model, objects are represented by physical particles on their contours. A simple notion of force generated by a linear actuator allows the model to create forces on each particle by way of a geometrical attractor. Tissue elasticity and contractility are modeled by local shape memory and muscular fibers attractors. A specific dynamic MRI study was used to build a simple trunk model comprised of by three compartments: lungs, diaphragm and abdomen. This model was registered on the real geometry. Simulation results were compared qualitatively as well as quantitatively to the experimental data, in terms of volume and geometry. A good correlation was obtained between the model and the real data. Thanks to this model, pathology such as hemidiaphragm paralysis can also be simulated.

Résumé. Dans cet article nous présentons un modèle discret 3D permettant de modéliser les mouvements du tronc pendant la respiration. Les objets du modèle sont représentés par des particules physiques sur leurs contours. Une notion simple de force induite par des actuateurs linéaires permet de génerer des forces au niveau des particules en utilisant un attracteur géométrique. Les propriétés élastiques et contractiles d'un tissu sont ainsi modélisées par des attracteurs de mémoire de forme locale et de fibre musculaire. À partir d'une étude spécifique en IRM dynamique, nous avons construit un modèle de tronc simplifié comprenant trois compartiments : les poumons, le diaphragme et l'abdomen. Ce modèle est recalé sur la géométrie réelle. Nous confrontons les simulations obtenues aussi bien qualitativement que quantitativement, en terme de variation de volume et de géométrie. Une bonne correlation est obtenue entre le modèle et les données réelles. Grâce à ce modèle nous montrons enfin que l'on peut simuler la paralysie hémidiaphragmatique.
\end{abstract}

\section{INTRODUCTION}

The diaphragm has two main roles: anatomically it separates the thoracic compartment from the abdominal compartment and physiologically it is the main respiratory muscle. The action of this muscle is complex and depends mainly on its size, its shape, and its attachments and links to surrounding organs and skeleton. The human adult diaphragm is shaped like a dome: a central tendon originates the muscular fibers. Laterally the fibers are inserted on the 7th to the 12th ribs (see Fig. 1, left). During inspiration, the diaphragm contracts and the abdominal content plays the role of a lever resulting in an enlargement of the thoracic cavity. This enlargement generates a negative pressure inside the rib cage, drawing air into the lungs. When the diaphragm relaxes, the air is expelled, helped also by the elasticity of the lung and the tissues lining the thoracic cavity. The

* This project was partly funded by CNRS ACINIM LePoumonVousDisJe and CNRS Inter-EPST program Bio-Informatique.

1 TIMC-IMAG, CNRS UMR 5525, Université Joseph Fourier, Grenoble, Institut d'Ingénierie de l'Information de Santé, Domaine de la Merci, F-38706 La Tronche Cedex, France. e-mail: Emmanuel.Promayon@imag.fr Pierre.Baconnier@imag.fr

2 CHU Grenoble, Hopital Michallon, F-38700 La Tronche, France

(C) EDP Sciences, SMAI 2008 

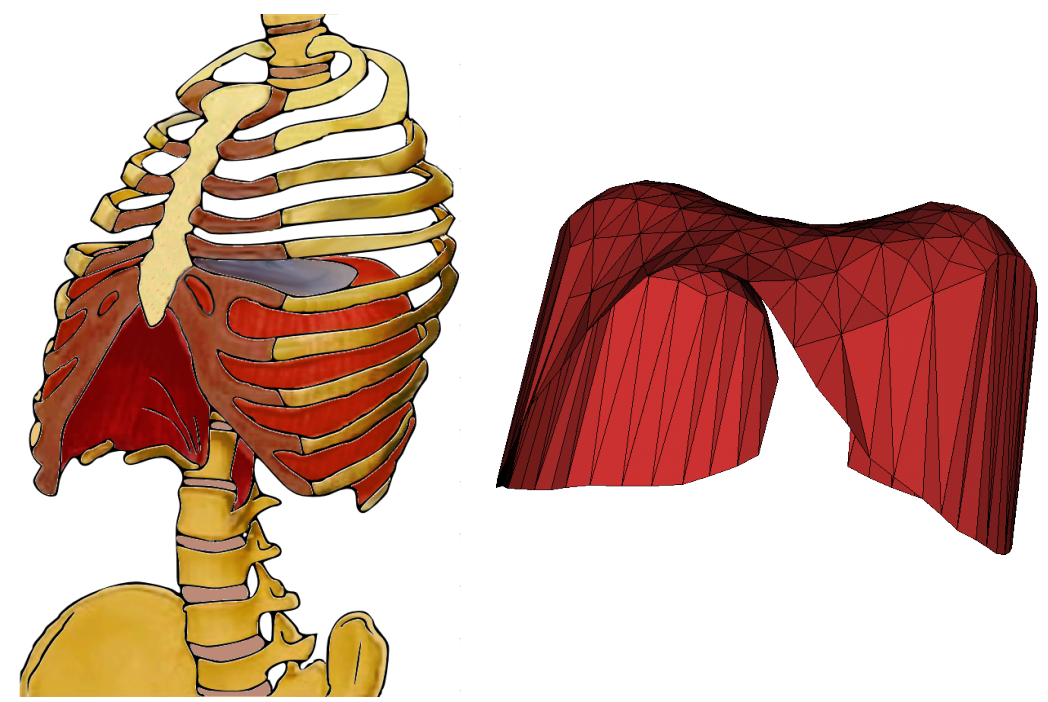

FIGURE 1. Human trunk. The diaphragm and its skeleton attachment (left). Reconstructed diaphragm surface (right).

abdominal compartment can be considered as incompressible during a given period of time (several minutes). Indeed, apart from a small gastric gas content, the abdominal cavity is filled with organs of quasi constant volume (blood volume variations are neglected) as all human tissues except lung. The stomach is commonly isolated from the remaining digestive tract by two closed sphincters, its gas content is then constant and considered incompressible in the range of observed gastric pressures.

A model of the diaphragm and its surrounding structures can be used in two simulation fields: physiology and computer assisted surgery. It has to be geometric and kinematic as well as dynamic. If the simulated movements are produced by the model at a sufficiently fast rate, it can be used to predict the diaphragm and abdominal organ positions during respiration thus being able to drive an imaging device or a conformative radiotherapy. It is also important to be able to model specific diaphragm pathologies, such as hemidiaphragm paralysis, as they can highly alter the abdominal organ movements.

Physiological studies of the respiratory system classically include volume and pressure variations. But as the diaphragm is not visible nor easily accessible from outside the body, studying the diaphragm deformation requires to use three dimensional medical images [21], either Computerized Tomography (CT) scan or Magnetic Resonance Imaging (MRI). Pettiaux and al [16] showed that CT scan allows satisfying 3D reconstructions of the diaphragm. Cluzel and al [5] and Craighero and al [7] shown similar results using MRI. There are few works dedicated to model the diaphragm muscle. Boriek and al. [3] used a Finite Element Method (FEM) membrane model to study the material behaviors but did not try to compare the model with experimental deformations. Kinetic modeling was also proposed in [12] and [15], using geometrical change to describe muscular actions. In respiratory physiology, the most famous model is a compartmental model where the rib cage and abdomen form two compartments and where an electric schema analogy is used to display the relationships between active and passive links. However it seems difficult to use this model to establish links between a given pathology and some local mechanical problems and to give 3D geometric information. Improvements of this model had recently been used to include planar geometry information [2]. Other models include computer graphics model, such as [23] and [24], and focus on computer graphics 3D animation rather than physiological realism. 


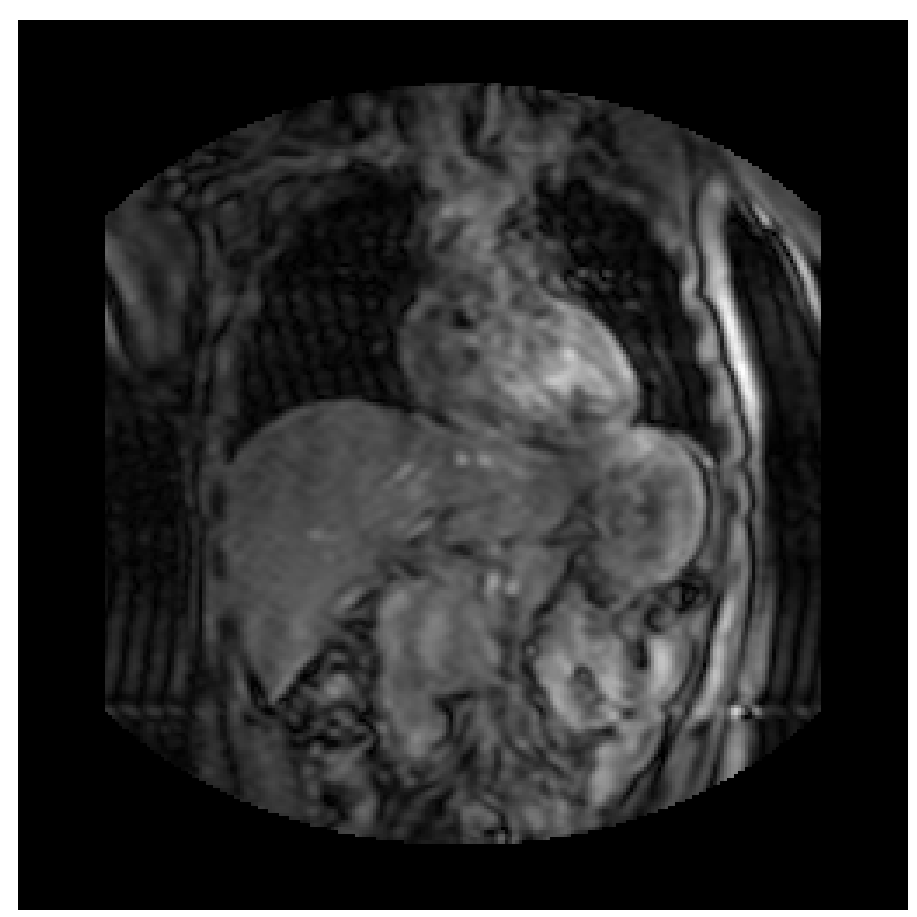

FIgURE 2. An MRI image acquired using the Fast Field Echo-Echo Planar Imaging. The resolution is $256 \times 256$ pixels. Note the variation of contrast, and the artifacts due to the acquisition velocity.

\section{Method}

\subsection{Available data}

Our current work is based on data acquired by a specific acquisition protocol [7]. A 1.5 Tesla MRI acquisition was performed using the Fast Field Echo-Echo Planar Imaging techniques. In conventional MRI each image is acquired in 5 seconds, has a resolution of $512 \times 512$ pixels and a thickness of $1 \mathrm{~mm}$. In [7], the acquisition time was reduced to $227 \mathrm{~ms}$ in order to study the diaphragm deformations. The main drawbacks of this technique are the image resolution $(256 \times 256$ pixels $)$, the slice thickness $(10 \mathrm{~mm})$ and the poor quality of the image due to movement and reconstruction artifacts (see Fig. 2 for an example of the acquired MRI data). Recently, using an enhanced protocol, MR images of three ventilated subjects were acquired. The respiratory volume and its variations were directly controlled by using artificial ventilation. From the MRI images, a post-synchronization process made possible the reconstruction of diaphragmatic surfaces (see Fig. 1, right).

\subsection{Model}

As presented in the previous paragraph, the only available data are poor quality MRI images (see Fig. 2). This yields to very strict specifications for the geometry and paramaters registration of the model. Another requirement for the model, as stated in the introduction, is its ability to produce very fast and accurate simulation. Two different directions can be taken by researchers to model human soft tissues [9]: the classical biomechanical approach and the computational discrete approach. The classical biomechanical approach is mainly based on the Continuum Mechanics or uses its counterpart, the FEM. It offers the advantage of being based on a strong theoretical background. There are generally two kinds of drawbacks when one applies this method to computer aided medical or physiological simulation: the computation time cost and the difficulties 
to build complex assemblage where rigid, elastic and active structures are interacting. Computation time can be reduced in this context, even for material with non-linear constitutive law, by using recent derived methods such as $[6,8,22]$ or alternative continuous models such as $[1,10,13,20]$. Finite Element Analysis is extremely interesting when one needs to understand and to know the consequences of a local deformation on the material stress and strain. However, in the present work, the aim is mainly to get an accurate, patient-specific geometry and dynamics. We need to know the consequences of the diaphragm contractions in terms of body structure displacements and deformations, i.e. at a higher scale than the many different materials composing the different organs and tissues. In the FEM, the extraction of physical parameters, such as Young modulus and Poisson ratio for linear constitutive law, is possible by measuring isolated tissue samples [11]. In vivo tissue characterization is essential because the mechanical behavior of soft tissues can differ significantly between in-vivo and exvivo conditions. Tissue characterization, as done in [14,19], is nevertheless extremely difficult to perform on living tissue and/or in vivo, notably due to the tissue accessibility, the organ movements or deformations, and the need to sterilize the measurement devices. In this study the in-vivo measurement of the tissues rheology is impossible as no direct access to the organs is provided. Moreover the muscle activation function are not anyhow available. This means that a global optimisation process using the whole organ geometries and deformations during respiration has to be enough to fit the geometry and the physical parameters of the model.

Our aims are to include multiple dynamic interactions and properties, to be able to produce real-time simulations, and to be able to fit the model only using the available MRI data. These aims justify the choice of a discrete approach. Previous works from the authors [17,18], and more recently from Zordan and al. [23, 24], used the same approach to build a visual simulation of the respiration. But in both cases, the simulation were not compared to patient-specific data nor even to experimental data. In this paper we propose to qualitatively and quantitatively compare the results of our discrete model simulation with the available MRI data.

To model living structures, we mainly need three different kinds of components:

- rigid components (to model skeleton),

- deformable components (to model soft tissues),

- and active deformable components (to model muscles).

In our model, these components are all derived from the same principle: a set of particles control the component surfaces, themselves organized using triangular facets. Each particle has a position, a mass and different properties depending on the kind of component it is part of. Accordingly, the particles in deformable components have an elastic property and the particles in active deformable components also have a contractile property. In essence, this is similar to a mass-spring network, but the elasticity is described using an original formulation, which allow better stability and control than mass-spring network, as shown in [18].

\subsection{Dynamics}

Forces are exerted on the particles to generate displacements and deformations. Three kind of forces are needed :

Force field: this kind of force is applied to all particles. At each time the force intensity and direction is known. This kind of force can vary depending of some mechanical or physical properties, e.g. the mass or the velocity of the particle. The gravitional force is an example of such a force.

Focal force: this is a kind of force known in intensity and direction and applied at specific time of the simulation. For example it can be a force applied to a particle by one of its neighbor in a particular type of interaction. This kind of force is also used to apply boundary conditions or to transmit user interaction in the model.

Linear actuator force (LAF): this kind of force depends on the internal state of the object, i.e. mainly on the geometry of neighboring particles. The intensity and direction of this kind of force is computed using local geometric or mechanical data and is generally updated at each time step. A LAF is used when a particle has to go toward an ideal position that minimizes a given function. Therefore, LAF are used to model elasticity and contracility. 


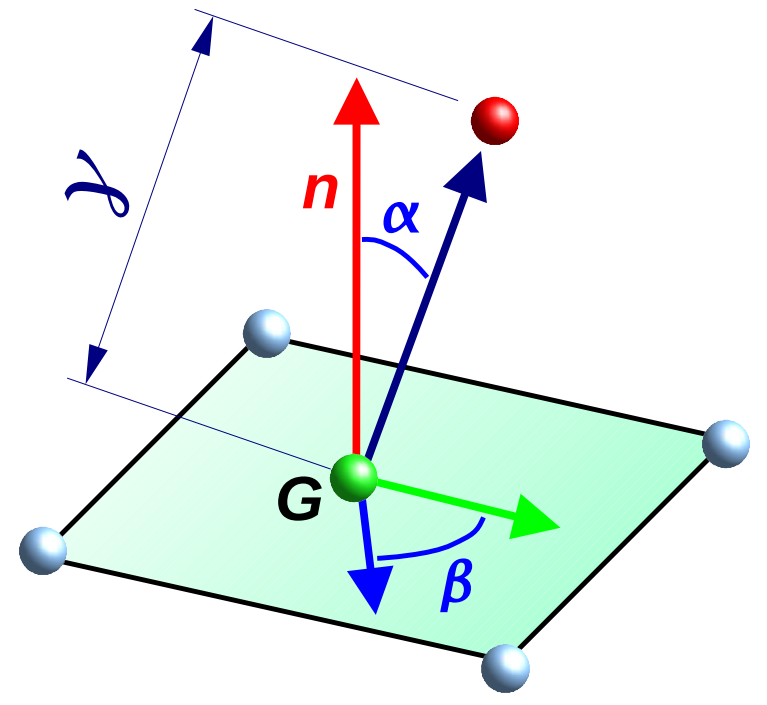

Figure 3. Local coordinate system use to define the shape function. For each particle three geometric parameters are used: $\alpha, \beta$ and $\gamma$. Once determined at rest shape, they can be use to target the position that minimize the deformation. The considered particle is in red, its neighbors are in blue, $G$ is the center of the neighbors masses, $n$ the approximated normal to the surface around the particle.

We introduce a LAF in order to minimize a given energy $E$. Whenever it is possible to define, for a given particle of 3D position $\mathbf{P}$, a position $\mathbf{P}_{\min }$ that is known to be minimizing $E$, a LAF can be used. Thus a LAF is simply a force that tends to minimize the distance $\left|\mathbf{P P}_{\mathbf{m i n} \mathbf{E}}\right|$. To express a LAF, we can use a simple expression such as:

$$
\mathbf{F}=k_{L A F}\left(\mathbf{P P}_{\min \mathbf{E}}\right)
$$

where $k_{L A F}$ is a parameter of the particle, or of a whole components. LAFs can thus be seen as potential forces that tends to minimize a distance. LAFs can model any kind of forces that could be defined by a target position. $\mathbf{P}_{\min }$ can depend on geometry or on constraints. The most important and difficult part is to determine a correct expression for $\mathbf{P}_{\mathbf{m i n}}$, so that it approximates a local minimum of $E$.

Spring-mass network parameters are known to be difficult to find and adjust. Therefore, our model does not use a network of springs to link the particles. To model the elastic property of a particle we define a local elasticity memory [17]. The elastic property of a particle is simply its ability to come back to its original geometric configuration once deformed. To model this property each particle has a local coordinate system defined relatively to its neighboring particles. This local coordinate system is defined by three parameters: two angles $\alpha$ and $\beta$ and one distance $\gamma$ [17], see Fig. 3. These three scalars are initialized at the rest configuration and are called $\alpha_{0}, \beta_{0}$ and $\gamma_{0}$. At any time, if a particle position verifies $\alpha=\alpha_{0}, \beta=\beta_{0}$, and $\gamma=\gamma_{0}$, then the particle is at the rest configuration, thus the component is locally undeformed. Using this local coordinate system, we can compute at any time and for each particle a position using $\alpha_{0}, \beta_{0}, \gamma_{0}$ and the position of neighboring particles. This position is ensured to locally minimize the deformation energy. Using this position as $\mathbf{P}_{\mathbf{m i n}}$ allows us to define a LAF to minimize this energy.

An elastic component is defined by a contour where this particular LAF is applied to all the particles. The elasticity parameter is the stiffness $k_{\text {elasticity }}$ used by the LAF.

Another LAF is used to model contractility. Once the contraction directions (muscular fibers) on the muscular component are defined, the position $\mathbf{P}_{\mathbf{m i n}}$ of a particle at one extremity of a contractile components can 
be simply defined as being the particle position at the other extremity of the fiber. The LAF could then be activated by varying the $k_{\text {contractile }}=A(t) \cdot k_{\text {muscle }}$ coefficient during the simulation. $k_{\text {muscle }}$ is constant. In order to activate the muscle contraction, $A(t)$ mimics a muscle activation signal. $A(t)$ can take all the values between [0..1]. When $A(t)=1$ the activation is maximal, and when $A(t)=0$, it is null.

To solve the system dynamics, at each time $t$, internal and external forces are computed, and the equation of motion are integrated, taking into account the local and global constraints.

Note that a particle mesh can include any types of particle. For example, it is possible to have an elastic particle with muscular neighbors. This does not generate any computational problem. Each particle accumulates its internal forces (elastic forces or muscular forces) and corresponding reaction forces are then distributed to its neighbors, in order to verify Newton's second law, independently of their types.

All the geometry and physical parameter are described using the PML language [4].

\subsection{Constraints and loads}

Forces are not often sufficient to model complex behaviors. Constraints are added to maintain some conditions like non-penetrating area or incompressibility. Our algorithm considers constraints as non-quantified force components: they are solved using a direct projection algorithm based on the gradient vector of the constraint function.

Volume preservation. It is possible to handle the total incompressibility of a closed contour, and therefore to have a tighter link with real tissues. Improvements of the previously published method (see [17]) allows for real-time computation of this particular constraint and thus for any kind of triangulated surface.

Volume preservation is an essential property of soft tissue modeling. The control of the volume is necessary in order to simulate both the incompressibility of some organs and to control the volume variation of other organs.

Consider one object described by a triangular mesh of particles at the contour, in our model, the volume preservation constraint is applied to all these particles. Note that the triangular mesh can also be used for visualization. Let $n$ be the number of particles of this triangular mesh. Let $\mathbf{P}_{\mathbf{i}}$ denotes the positions of the $n$ particles. Let $V_{0}$ be the initial (rest shape) volume of the mesh and $V\left(\mathbf{P}_{\mathbf{1}}, \cdots, \mathbf{P}_{\mathbf{n}}\right)$ a function of the particle positions that gives the current volume value. If the volume-controled mesh is deformed during the simulation, our algorithm provides a fast and efficient way to preserve the inner volume while keeping the mesh shape similar. Let $\hat{\mathbf{P}}_{i}, i \in[1 \cdots n]$, be the positions of the particles before the correction due to the volume constraint, that is to say just after the model forces have been summed and integrated on each particles. Our method is able to find the displacements to apply to each particle in order to correct the current volume. In order to find these displacements, the following system has to be solved:

$$
\left\{\begin{aligned}
\mathbf{P}_{i} & =\hat{\mathbf{P}}_{i}+\lambda \frac{\partial V}{\partial \hat{\mathbf{P}}_{\mathbf{i}}}, \forall i \in[1 \cdots n] \\
V\left(\mathbf{P}_{\mathbf{1}}, \cdots, \mathbf{P}_{\mathbf{n}}\right) & =V_{0}
\end{aligned}\right.
$$

where $\mathbf{P}_{i}$ are the corrected positions and $\lambda$ is the unknown scalar. $\lambda \frac{\partial V}{\partial \hat{\mathbf{P}}_{\mathbf{i}}}$ is equivalent to a constrained corrective displacement that solve the volume-preservation constraint. Solving system (2) allows us to directly find a solution for the volume-preservation problem. By rearranging the equations, we can simplify system (2) into an equation in $\lambda^{3}$ which coefficients depend only on $\hat{\mathbf{P}}_{\mathbf{i}}$ and $\lambda \frac{\partial V}{\partial \hat{\mathbf{P}}_{\mathbf{i}}}$. Compared to lagrangian methods (lagrangian multiplier and minimization algorithm), our method exactly solves the constraint and is very fast as it is mainly the direct solution of a third degree equation. Note that this algorithm can also be used to control the volume variation of an object by modifying the targeted $V_{0}$ during simulation. 

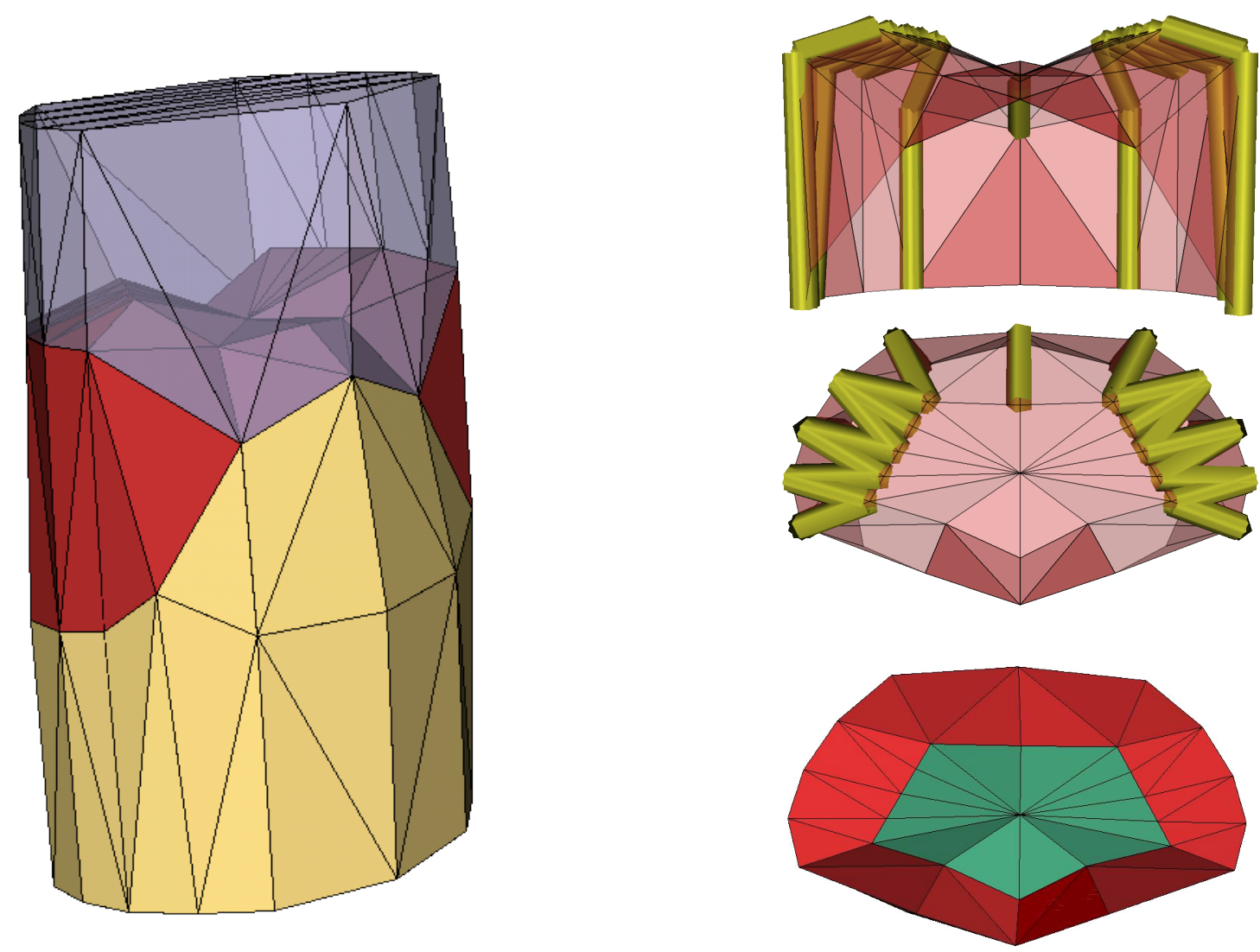

Figure 4. The healthy diaphragm model. The complete model using three compartments (lungs, diaphragm and abdomen) is shown on the left. The contraction fibers in the healthy diaphragm are represented as cylinders (top right). The central tendon of the diaphragm is shown on the bottom right.

Boundary conditions. On top of all forces and the volume preservation constraint, any other boundary conditions can be applied, such as null or imposed displacement in any direction, and imposed forces. All this kind of boundary conditions are described using the LML language [4], allowing for a dynamic change of the boundary conditions if needed during the simulation.

\subsection{Healthy and pathological diaphragm models}

Our discrete modeling framework was used to describe a simplified human trunk (see Fig. 4, left). It includes three components: lungs, diaphragm and abdomen for a total of 113 particles. The lung is a passive area, and is only modeled geometrically to monitor the volume variation $\Delta V$ generated by the diaphragm contraction. The diaphragm is modeled using an elastic and contractile component. The abdomen is an elastic component. The model geometry was registered using an elastic matching algorithm to the geometry segmented and reconstructed from the MRI at the beginning of inspiration.

The muscular fibers are defined on the model by selecting the particle that mimic the real muscular fibers: their direction is vertical and along the zone of apposition (see Fig. 4, top right). The activation function is set to mimic the physiological signal (linear contraction for 2 seconds, important decrease for 0.5 seconds and then normal decrease for 2 seconds). To model the central tendon, we set $k_{\text {elasticity }}$ of the top central part of the diaphragm as being twice as rigid as the other diaphragm areas (see Fig. 4, bottom right). 


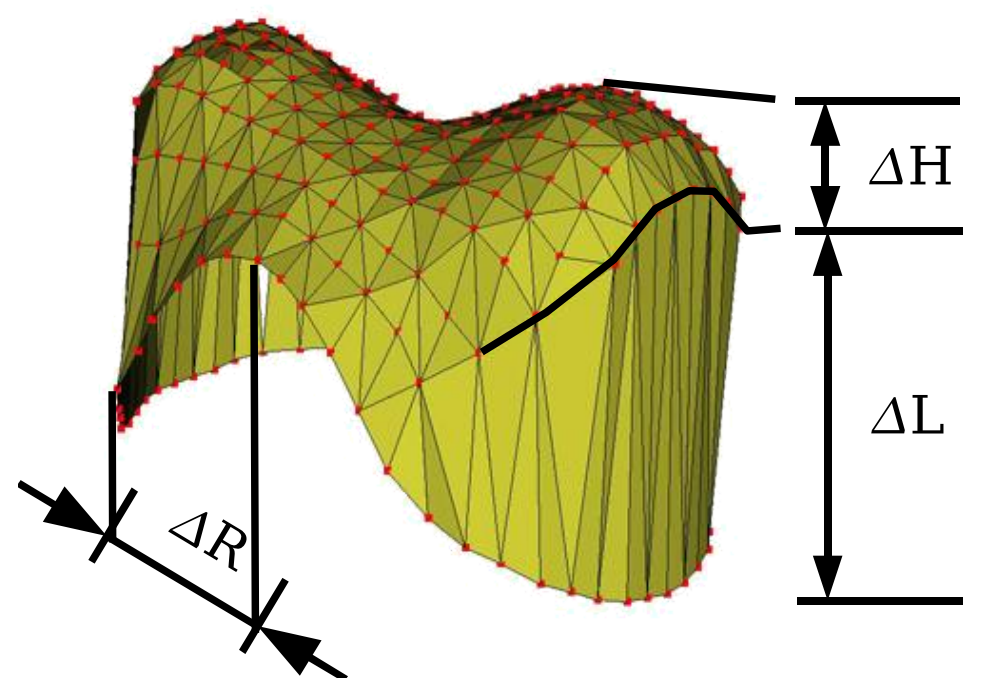

Figure 5. Comparison measurements. Deformations are measured in the zone of apposition $(\Delta L)$, the height of the diaphragmatic domes $(\Delta H)$ and the transverse direction $(\Delta R)$.

As the abdominal compartment can be considered as incompressible, the boundary conditions essentially consist in maintaining the incompressible constraint on the mesh defined by the diaphragm and the abdomen walls. A null displacement boundary condition is imposed to some particles to model the spine, the pelvis, and the top of the lung.

The model was compared with the experimental data by studying the deformation during a respiratory cycle in different directions (Fig. 5). We compared deformation in terms of the variations of, from the most significant to the least significant: pulmonary volume $(\Delta V)$, apposition zone length $(\Delta L)$, height of the diaphragmatic domes $(\Delta H)$, and transverse length $(\Delta R)$.

We also simulated a pathological condition: an hemidiaphragm paralysis. This was obtained by inactivating all fibers of the same side of the diaphragm. An additional modification was needed: the pathological model has to include the long term effect of the paralysis, namely the elasticity loss of the paralysed hemidiaphragm. We thus set a different value of the elasticity parameter $k_{\text {elasticity }}$ for half of the diaphragm tissue. This pathological situation is known to lead to many ventilatory impairments among which a drastic decrease of inspiratory muscle efficiency, inducing a decrease of tidal volume (total volume displacement of each breath) and a paradoxical upward ("expiratory") movement of the paralysed hemidiaphragm during inspiration.

\subsection{Estimation of the model parameters}

In order to estimate the model parameters, the only available data were the pulmonary volume variation $\Delta V$. The main advantage of our model is its very fast computation time and its reduced number of parameters. In the trunk model, only two parameters are to be estimated: $k_{\text {elasticity }}$ and $k_{\text {muscle }}$. An optimization algorithm based on an "analysis by synthesis" strategy was elaborated. It consisted in a four step loop: (1) assume a given set of parameters, (2) build and simulate a respiratory cycle using the model, (3) compare the provided simulations with the respiratory volume measurements in the least square sense, (4) from this comparison deduce better values of parameters in order to improve the simulation/measurement fit. This loop was continued until the comparison carried out in (3) gives satisfactory results. 


\begin{tabular}{lcccccccc}
\hline & \multicolumn{10}{c}{ Volume and measurements } \\
\cline { 2 - 9 } & $\begin{array}{c}\text { End expiratory } \\
\text { vol. }(\mathrm{ml})\end{array}$ & $\begin{array}{c}\text { End inspiratory } \\
\text { vol. }(\mathrm{ml})\end{array}$ & $\begin{array}{c}\Delta V \\
(\mathrm{ml})\end{array}$ & $\begin{array}{c}\Delta V \\
(\%)\end{array}$ & $\begin{array}{c}\Delta L \\
(\mathrm{~mm})\end{array}$ & $\begin{array}{c}\Delta L \\
(\%)\end{array}$ & $\begin{array}{c}\Delta H \\
(\mathrm{~mm})\end{array}$ & $\begin{array}{c}\Delta R \\
(\mathrm{~mm})\end{array}$ \\
\hline Experimental data & 4760 & 5226 & 466 & 9.78 & 9.13 & 6.13 & 2.00 & 0.00 \\
Model & 4759 & 5197 & 438 & 9.20 & 9.73 & 5.98 & 13.30 & 8.80 \\
\% error & -0.02 & -0.55 & -6 & & 6.57 & & & \\
\hline
\end{tabular}

TABLE 1. Qualitative comparisons between real data and model.
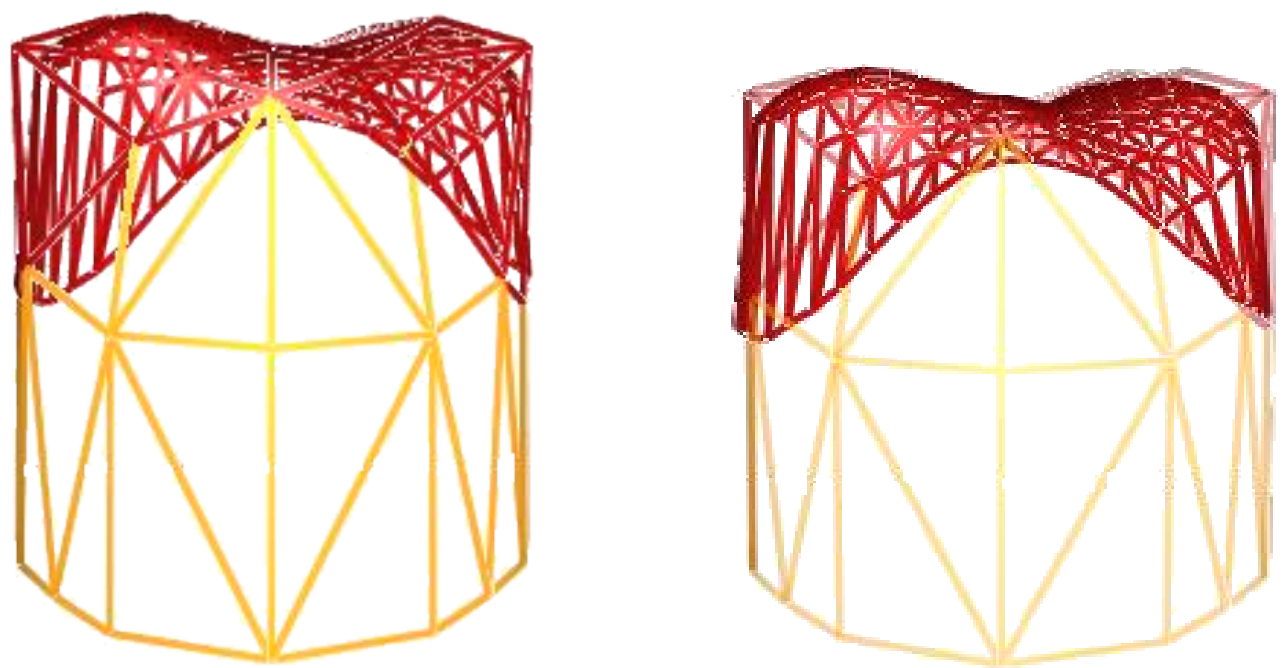

FiguRE 6. Comparisons of the model diaphragm geometry (coarse mesh) and reconstructed diaphragmatic surface. The model was initially deformed to match the reconstructed shape at beginning of the respiratory cycle (end expiratory position) (left). After the simulation of a respiratory cycle, the simulated deformation are superimposed with reconstructed diaphragmatic surface at the end of inspiration (right).

\section{Results}

The simulation of a complete respiratory cycle only takes 1.50 seconds on a Pentium Xeon 5140 at $2.33 \mathrm{Ghz}$, i.e. a frame rate of approximately 3000fps. Comparisons between the model and the real data are presented in Table 1. Qualitative geometry comparisons were also made between the surface of the diaphragm in the model and the reconstructed diaphragmatic surface at the end of inspiration (see Fig. 6).

The model is able to simulate the hemidiaphragm paralysis pathology. Comparisons between healthy and pathological diaphragm can easily be observed in 3D (see Fig. 7).

We also can see significant differences between lung volume displacements (see Fig. 8).

\section{Discussion}

\subsection{Healthy diaphragm}

The model was able to reproduce an accurate volume variation $\Delta V$ and piston-like deformation $\Delta L$. The model deformation compared to real deformation measured on a subject was as well qualitatively satisfying. Another very important point, especially when considering the application of the method in computer assisted 

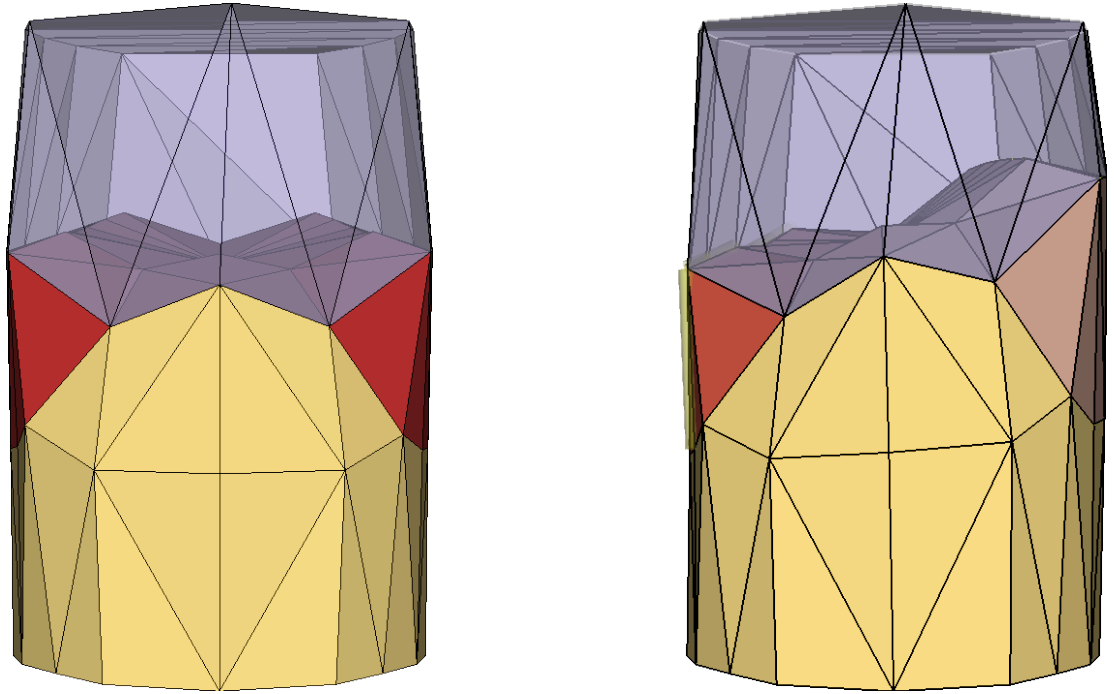

FiguRE 7. Comparison between a healthy diaphragm model and hemidiaphragm paralysis model using the same activation function at end inspiration (during exercise breathing).

medical intervention and physiological studies, is the fast computation time: the simulation is about four times faster than the respiratory cycle it is simulating. This result leaves some space for the improvement and enhancement of our model.

On the other side the simulation are far from correct when $\Delta H$ and $\Delta R$ are compared. These differences are probably due to an over simplification in the discretization of the diaphragmatic zone and to the model itself, which does not take contact and friction into account.

Although discrete and very simple, this model efficiently reproduced the complex movements of breathing. The major drawback of this model is that being discrete, it is not possible to compute the extract strain and stress on the different components. As these values on the in vivo diaphragm are not obtainable by any technique, the choice of a continuous model does not seem to be crucial.

\subsection{Hemidiaphragm}

We observed all the clinical consequences of the simulated pathology. The paradoxical behaviour of the paralysed hemidiaphragm (upward displacement during inspiration) is evidenced on the 3D simulation (Fig. 7, right). The difference between volume displacements in the healthy and pathological diaphragm amounts to what is typically measured (a $50 \%$ shortening, T. Similowski, personal communication) in clinical results (Fig. 8).

\subsection{Future works}

Once the $k_{\text {elasticity }}$ parameter is set for a given subject, the main advantage of this optimization technique is that we can directly and quickly adjust $k_{\text {muscle }}$ depending on $\Delta V$. This can lead to a real-time prediction of the diaphragm position during breathing, considering only one medical image at the beginning from which the diaphragm geometry can be registred. This could be used for example during conformative radiotherapy. Future works on the diaphragm model will include testing and validating other breathing situations and comparisons with other subject data. To overcome the differences noted for $\Delta H$ and $\Delta R$ we also plan to add the rib cage and its cartilage components (this work has just started, see Fig. 9). 


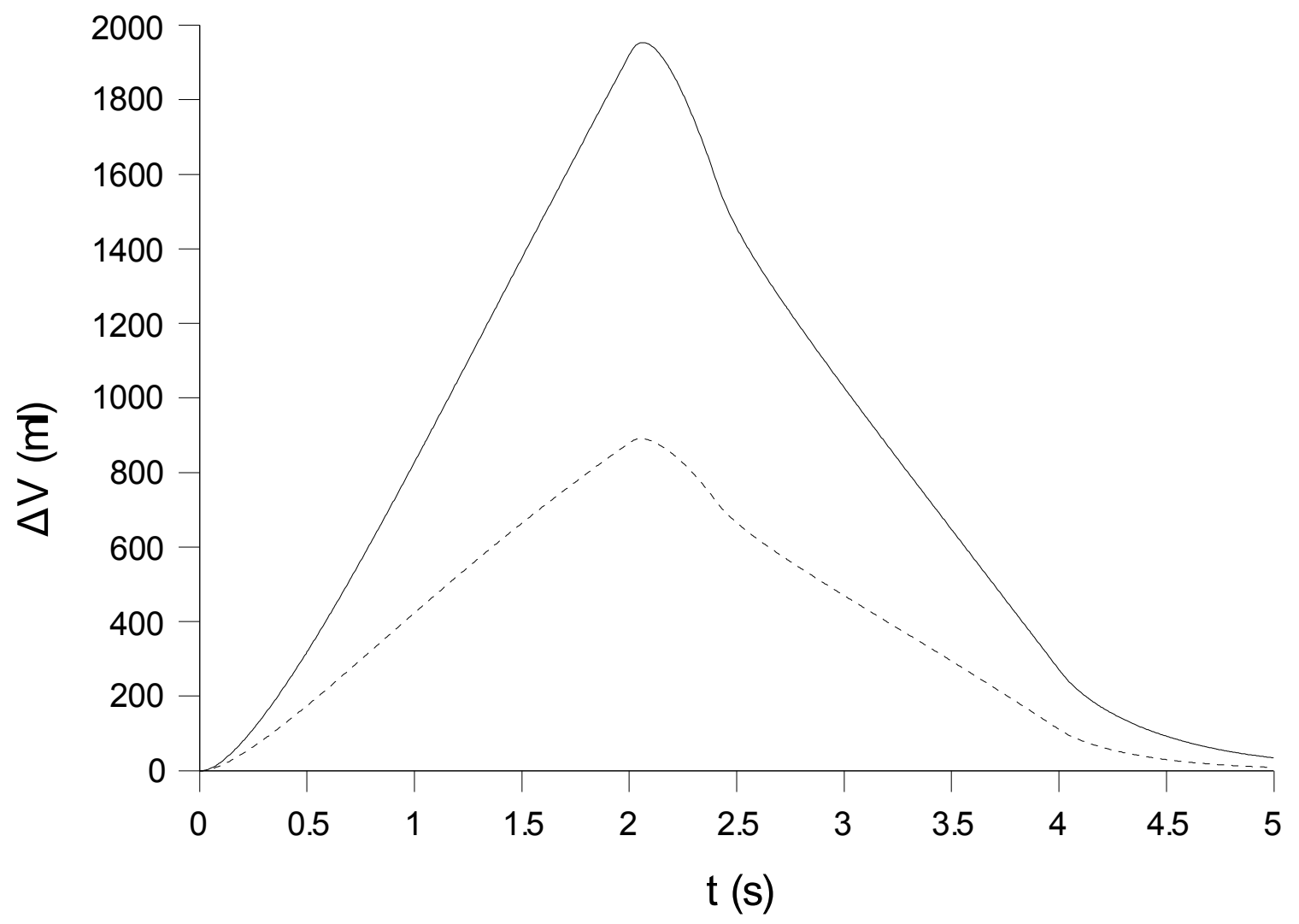

Figure 8. Comparison of the volume variation during exercise breathing generated by the healthy diaphragm model (continuous line) and the hemidiaphragm paralysis model (dashed line).

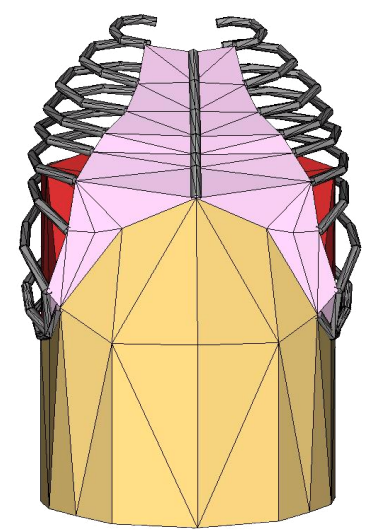

Figure 9. Advanced model of the human trunk including one solid body per rib with elastic links to model cartilaginous tissues. 


\section{ACKNOWLEDGEMENT}

The authors wish to thank L. Gaillard for her contribution to this work, and T. Similowski for suggesting the simulation of the hemidiaphragm paralysis.

\section{REFERENCES}

[1] Jernej Barbič and Doug L. James. Real-time subspace integration for St. Venant-Kirchhoff deformable models. ACM Transactions on Graphics (SIGGRAPH 2005), 24(3):982-990, August 2005.

[2] S. Basso-Ricci, P. Cluzel, A. Constantinescu, and T. Similowsky. Technical note - mechanical model of the inspiratory pump. J Biomech, 35(1):139-145, January 2002.

[3] A. M. Boriek and J. R. Rodarte. Effects of transverse fiber stiffness and central tendon on disaplacement and shape of a simple diaphragm model. J. Appl. Physiol., 82(5):1626-1636, 1997.

[4] M. Chabanas and E. Promayon. Physical model language: Towards a unified representation for continuous and discrete models. In International Symposium on Medical Simulation, volume 3078 of Lecture Notes in Computer Science, pages 256-266. Springer Verlag, 2004.

[5] P. Cluzel, T. Similowsky, C. Chartrand-Lefebvre, M. Zalter, J. P. Derenne, and P. A. Grenier. Diaphragm and chest wall: Assessment of the inspiratory pump with $\mathrm{mr}$ imaging - preliminary observations. Radiology, 215(2):574-583, 2000.

[6] S. Cotin, H. Delingette, and N. Ayache. Real-time elastic deformations of soft tissues for surgery simulation. IEEE Transactions On Visualization and Computer Graphics, 5(1):62-73, January-March 1999.

[7] S. Craighero, E. Promayon, P. Baconnier, J. F. Lebas, and M. Coulomb. Dynamic echo-planar mr imaging of the diaphragm for a 3d dynamic analysis. European Radiology, 15:742-748, April 2005.

[8] Gilles Debunne, Mathieu Desbrun, Marie-Paule Cani, and Alan H. Barr. Dynamic real-time deformations using space \& time adaptive sampling. In SIGGRAPH '01: Proceedings of the 28th annual conference on Computer graphics and interactive techniques, pages 31-36, New York, NY, USA, 2001. ACM.

[9] H. Delingette. Towards realistic soft tissue modeling in medical simulation. IEEE Special Issue on Virtual and Augmented Reality in Medicine, 86(3):512-523, 1998.

[10] H. Delingette, S. Cotin, and N. Ayache. Efficient linear elastic models of soft tissues for real time surgery simulation. In Medecine Meets Virtual Reality VII, Interactive Technology and the New Paradigm for He althcare, pages 139-151. IOS Press, January 1999.

[11] Y. C. Fung. Mechanical Properties of Living Tissues. Springer Verlag, 2nd edition edition, 1993.

[12] A. P. Gauthier, S. Verbank, M. Estenne, C. Segebarth, P. T. Macklem, and M. Paiva. Three-dimensional reconstruction of the in vivo human diaphragm at different lung volumes. J. Appl. Physiol., 72(4):1407-1412, 1994.

[13] Doug L. James and Dinesh K. Pai. Multiresolution green's function methods for interactive simulation of large-scale elastostatic objects. ACM Trans. Graph., 22(1):47-82, 2003.

[14] A. Nava, E. Mazza, M. Furrer, P. Villiger, and W.H. Reinhart. In vivo mechanical characterization of human liver. Medical Image Analysis, 12(2):203-216, April 2008.

[15] M. Paiva, S. Verbank, M. Estenne, C. Segebarth, and P. T. Macklem. Mechanical implications of in vivo human diaphragm shape. J. Appl. Physiol., 72(4):1407-1412, April 1992.

[16] N. Pettiaux, M. Cassart, M. Paiva, and M. Estenne. Three-dimensional reconstruction of the human diaphragm with the use of spiral computed tomography. J. Appl. Physiol., 82(3):998-1002, 1997.

[17] E. Promayon, P. Baconnier, and C. Puech. Physically-based deformations constrained in displacements and volume. Computer Graphics Forum, Eurographics96, 15(3):155-164, August 1996.

[18] E. Promayon, P. Baconnier, and C. Puech. Physically-based model for simulating the human trunk respiration movements. In CVRMed II - MRCAS III, volume 1205 of Lecture Notes in Computer Science, pages 379-388. Springer Verlag, 1997.

[19] E. Samur, M. Sedef, C. Basdogan, L. Avtan, and O. Duzgun. A robotic indenter for minimally invasive measurement and characterization of soft tissue response. Medical Image Analysis, 11(4):361-373, August 2007.

[20] J. Teran, S. Blemker, V. Ng Thow Hing, and R. Fedkiw. Finite volume methods for the simulation of skeletal muscle. In $S C A$ '03: Proceedings of the 2003 ACM SIGGRAPH/Eurographics symposium on Computer animation, pages 68-74, Aire-la-Ville, Switzerland, Switzerland, 2003. Eurographics Association.

[21] W. A. Whitelaw. Shape and size of the human diaphragm in vivo. J. Appl. Physiol., 82(3):998-1002, 1987.

[22] Wen Wu and Pheng Ann Heng. A hybrid condensed finite element model with gpu acceleration for interactive 3d soft tissue cutting: Research articles. Comput. Animat. Virtual Worlds, 15(3-4):219-227, 2004.

[23] Victor Brian Zordan, Bhrigu Celly, Bill Chiu, and Paul C. DiLorenzo. Breathe easy: Model and control of simulated respiration for animation. In SCA '04: Proceedings of the 2004 ACM SIGGRAPH/Eurographics Symposium on Computer Animation, pages 29-37, Aire-la-Ville, Switzerland, Switzerland, 2004. Eurographics Association.

[24] Victor Brian Zordan, Bhrigu Celly, Bill Chiu, and Paul C. DiLorenzo. Breathe easy: model and control of simulated respiration for animation. Graphical Models, 68(2):113-132, March 2006. 